\title{
Reversal of a visual illusion of length perception
}

\author{
W. H. TEDFORD and C. F. GRAY \\ Southern Methodist University, Dallas, Texas 75275
}

\begin{abstract}
One-hundred and five human subjects compared an open interval, bounded by vertical lines, to one of equal length which contained an intervening dot. Open intervals were judged to be shorter when the lengths subtended a visual angle of less than approximately $9 \mathrm{deg}$, but longer when the visual angle was greater. There is a striking similarity to the perception of time intervals which contain intervening stimuli.
\end{abstract}

A simple illusion which has received a great deal of study throughout the history of psychology is the effect of intervening stimuli on the perception of a time interval. In general, intervening stimuli cause short intervals to be overestimated (Buffardi, 1971; Hall \& Jastrow, 1886) and long ones to be underestimated (Swift \& McGoech, 1925; Whitely \& Anderson, 1930). One might infer from the experiments of Obonai (1954) that a similar reversal would occur in the perception of open vs. closed, or interrupted, distance. The present study attempted to find such a reversal.

\section{METHOD}

\section{Subjects}

Volunteers from undergraduate psychology classes were tested in groups of varying sizes. A total of 105 subjects participated.

\begin{abstract}
Apparatus
Intervals to be judged were marked off by vertical lines. They were presented in pairs. one above the other. with the lower interval offset to the right. Relative interval lengths were 1, 2. 4, 8, and 16 . As projected on a screen. these were $7, .14,28.56$. and $112 \mathrm{~cm}$. A "closed" interval contained a black circle, $7 \mathrm{~cm}$ in diam. centered between the vertical lines. The lines marking the ends of the interval were $14 \mathrm{~cm}$ high. Viewing distance was approximately $4.5 \mathrm{~m}$.

There were 10 test pairs. each consisting of a closed and an open interval of equal length; half of the test pairs had the open interval at the top and half at the bottom. In addition. the five open intervals were each paired with themselves. A final eight pairs were of clearly unequal lengths. included to prevent the discovery that all pairs were equal.
\end{abstract}

\section{Procedure}

The 23 slides were randomized and presented for 3 sec each with $5 \mathrm{sec}$ between slides. The slide number was announced before each presentation. Subjects had a numbered answer sheet and responded by checking whether the upper or lower member of the pair appeared longer. A forced choice procedure was used: however. a few subjects did not respond to some slides.

\section{RESULTS}

Figure 1 indicates the percentage of times the closed interval was judged to be longer. Because of some failures to respond, each data point is based on between 200 and 209 judgments. Using a binomial

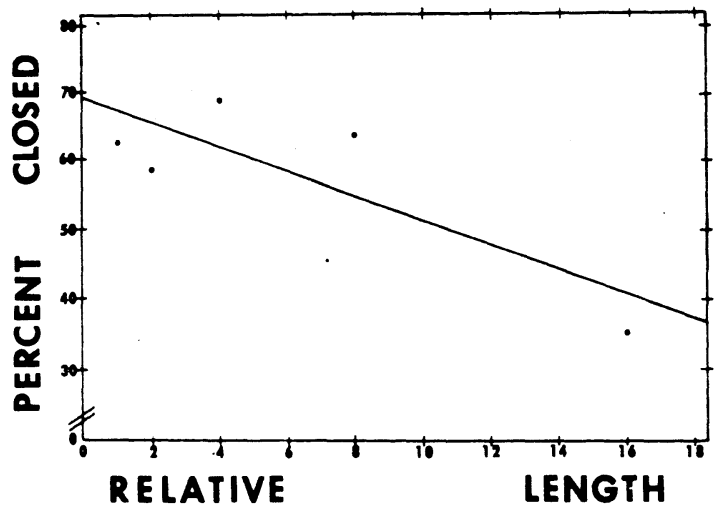

Figure 1. Percentage of trials on which the closed interval was judged to be longer than an open one of equal length.

test. all points are significantly different from $50 \%$ at the .02 level or greater.

The straight line represents a least-squares fit to the data $(Y=69-1.74 X)$ and indicates a cross-over point at approximately $9 \mathrm{deg}$ of visual angle. It is, of course, highly doubtful that the percentage is a linear function of interval length, but with only one point beyond the cross-over, this seems the best approximation. An additional 100 subjects were tested with a new set of slides which contained more long intervals. However, a number of the slides had photographic flaws. To the extent that the data were valid, they confirmed that the illusion does reverse. Data from 10 subjects tested individually at varying viewing distances also confirmed the reversal, as well as the fact that viewing distance influences the cross-over point. The closer the viewing distance, the shorter the length at cross-over, as would be expected.

There were no significant differences from $50 \%$ when the open intervals were paired with themselves. indicating no bias favoring the upper or lower stimuli. There were no sex differences.

\section{DISCUSSION}

It seems clear that the open vs. closed interval illusion reverses. dependent on the interval length being judged. This assumes that the size of the intervening stimulus remains constant. While Restle 
and Merryman's (1969) results would indicate that the height of the end markers may be a minor influence, the main parameters are probably interval length and size of the intervening stimulus.

The shift in the magnitude of the cross-over length with viewing distance indicates that it remains constant in terms of visual angle. This would imply some physiological basis for the reversal. On the other hand, the analogy to the reversal in time perception argues for a cognitive, or judgmental, explanation.

\section{REFERENCES}

BUFFARDI, L. Factors affecting the filled-duration illusion in the auditory, tactual, and visual modalities. Perception \& Psychophysics, 1971, 10, 292-294.
Hall, G. S., \& Jastrow, J. Studies of rhythm. Mind, 1886, $11,55-62$.

Oв ONAI, T. Induction effects in estimates of extent. Journal of Experimental Psychology, 1954, 47, 57-60.

Restle, F., \& MERRYMAN, C. Distance and an illusion of length of line. Journal of Experimental Psychology, 1969, 81. 297-302.

Swift, E. J., \& McGeoch, J. A. An experimental study of the perception of filled and empty time. Journal of Experimental Psychology, 1925, 8, 240-249.

Whitely, P. L., \& ANDERSON, J. C. The influence of two interpolations upon time perception. Journal of General Psychology, 1930, 4, 391-401.

(Received for publication September 29, 1975.) 